\title{
Phaeoacremonium species detected in fine needle aspiration: a rare case report
}

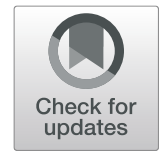

\author{
Santosh Tummidi ${ }^{1 *} \mathbb{D}$, Bitan Naik', Arundhathi Shankaralingappa', Pavithra Balakrishna², \\ Arati Ankushrao Bhadada ${ }^{3}$ and Navya Kosaraju ${ }^{4}$
}

\begin{abstract}
Background: Fine needle aspiration cytology (FNAC) with rapid on-site evaluation has a great potential for the diagnosis of fungal lesions and other opportunistic infections. Fungal infections have been in increasing trend in the past two decades due to immunosuppression, travel, and environmental exposure. Human disease caused by Phaeoacremonium species is rare and was first reported in 1974 as subcutaneous tissue infection in a renal transplant recipient.

Case presentation: We report a case of subcutaneous tissue swelling in a 67-year-old male, wherein FNAC was done with incidental detection of the fungus (Phaeoacremonium spp).

Conclusion: There are very few reported cases of subcutaneous infection in humans by Phaeoacremonium spp. Clinical suspicion and FNAC can play an important role in early detection of the fungus, prevent spread, and facilitating early treatment.
\end{abstract}

Keywords: Phaeoacremonium, Fungal, Toluidine blue, Fine needle aspiration, Rapid-on-site

\section{Background}

Phaeohyphomycosis is a rare form of sporadic infection that is caused by dematiaceous fungi. It's commonly detected in tropics and sub-tropical environments. It's a saprophyte in soil and vegetation causing Petri or esca diseases in plants $[1,2]$. The first reported case was in 1974 by Ajello et al in a renal transplant patient [3]. Most often, the diagnosis is delayed due to the rarity and variable nature of the presentation $[4,5]$.

FNAC can play a definitive role in early identification of the disease process [6]. A high index of clinical suspicion of fungal infections (phaeohyphomycosis) should always be considered in immunocompromised patients, specifically in patients with a history of diabetics, old age, travel to tropics for early treatment [4]. We report a

\footnotetext{
* Correspondence: born_vss@yahoo.co.in

'Department of Pathology, All India Institute of Medical Sciences, Mangalagiri, Andhra Pradesh 522503, India

Full list of author information is available at the end of the article
}

rare case of Phaeoacremonium spp. incidentally detected in FNAC and later confirmed by fungal culture.

\section{Case presentation}

A 67-year-old male daily construction labourer by profession, presented to surgical outpatient department with a subcutaneous nodule in the medial aspect of the right knee joint for 10 years. He had no significant history of any trauma. On examination the nodule was in a subcutaneous location with firm consistency, slightly tender, restricted mobility, not fixed to underlying structures and was measuring $4 \times 3 \mathrm{~cm}$ (Fig. 1a).

Laboratory investigations revealed random blood sugar - $452 \mathrm{mg} / \mathrm{dL}, \mathrm{HbA1c}$ value was $13 \%$ (estimated average glucose / eAG - $326 \mathrm{mg} / \mathrm{dL}$ ) (Normal HbA1c < 5.7\%). His hemogram, liver function and renal function tests was within normal limits. Rapid lateral flow assay tests for Human immunodeficiency virus, Hepatitis B, and Hepatitis $\mathrm{C}$ were negative. Plain X-ray of knee joint revealed no bony lesion (Fig. 1b). Ultrasonography of 


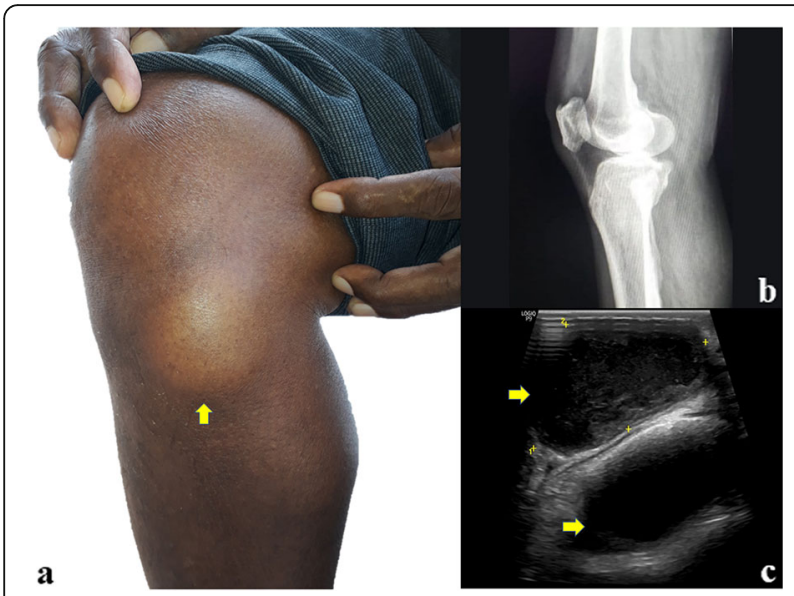

Fig. 1 a Clinical picture of patient showed a nodule in subcutaneous location below right knee joint in anteromedial aspect with firm consistency and measuring $4 \times 3 \mathrm{~cm}$ approximately. $\mathbf{b}$ X-ray revealed no bony involvement; c USG of medial aspect of right knee showed two well defined oval anechoic thick-walled lesions measuring $4.5 \times 2.6 \mathrm{~cm}$ and $4.0 \times 2.4 \mathrm{~cm}$ in the subcutaneous plane

medial aspect of right knee showed two well defined oval anechoic thick-walled lesions measuring $4.5 \times 2.6 \mathrm{~cm}$ and $4.0 \times 2.4 \mathrm{~cm}$ in the subcutaneous plane, below knee joint in anteromedial aspect. Colour Doppler shows no significant vascular uptake (Fig. 1c). A clinical suspicion of lipoma was made and sent for FNAC.

FNAC with Rapid On-Site Evaluation (ROSE) was done using toluidine blue using a 22-gauge needle and 5 $\mathrm{ml}$ syringe. Aspirate of $2 \mathrm{ml}$ pus-like material. ROSE of slide revealed the presence of negatively stained acute angle branched hyphae along with inflammatory background (Fig. 2). The slides were sent for routine stains.

Cytosmears showed plenty of inflammatory cells comprising of neutrophils, lymphocytes, histocytes, nuclear

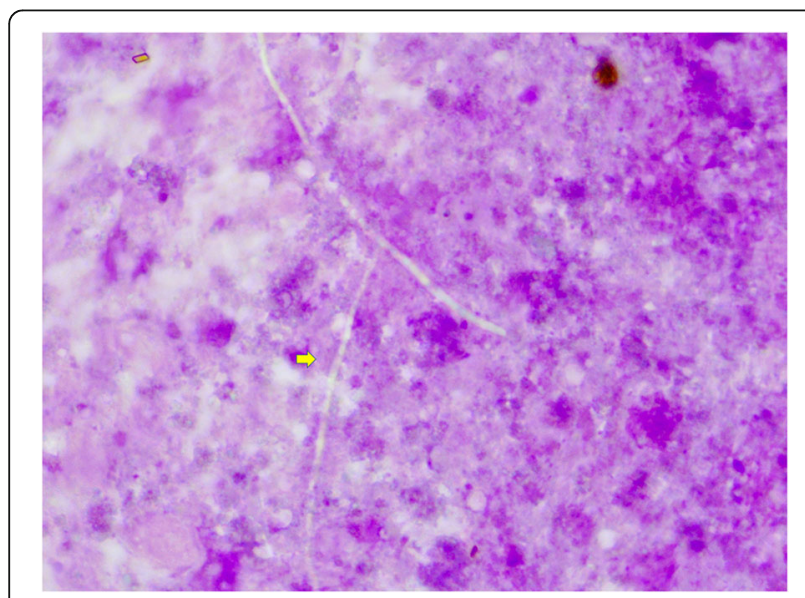

Fig. 2 Rapid onsite staining showing the presence of negatively stained acute angle branched hyphae (arrow) along with inflammatory background. (Tol Blue, $\times 100$ ) debris, and eosinophilic bodies / Splendore-Hoeppli phenomenon along with numerous fungal septate hyphae with acute angle branching (Fig. 3a,b). The fungal hyphae were positive for Periodic acid-Schiff (PAS) stain (Fig. 3c,d). Gram stain and Ziehl-Neelsen stain were negative. Cell block sample also revealed similar fungal branching hyphae with PAS positivity (Fig. 4a, b).

A diagnosis of inflammatory lesion possibly due to fungal aetiology was given on FNAC \& Cell block. The aspirated sample was sent for fungal culture. The fungal culture was done only on Sabouraud's dextrose agar (SDA) slant and incubated at $37^{\circ} \mathrm{C} / 25^{\circ} \mathrm{C}$. Growth on tube was noted on 3rd week of incubation. SDA slant showed surface greyish beige to black color and developed clusters of aerial hyphae (Fig. 4c).

Microscopic findings on Lactophenol cotton blue (LPCB) mount showed young hyphae with brownish appearance and side by side to form thick bundles (fascicles), cylindrical phialides along the hyphae and oblong conidia were present gathering in clusters at end of phialide (Fig. 4d). Hence a diagnosis of Phaeoacremonium parasiticum was given.

The patient was advised oral itraconazole $100 \mathrm{mg}$ BD for 1 month and currently under follow up with subsidence of the lesion.

\section{Discussion \& Conclusion}

Phaeohyphomycosis an opportunistic mycotic infection caused by an heterogeneous group of phaeoid pigmented fungi (Black mold) found widely disseminated in nature $[4,7]$. Morphologically these dematiaceous fungi have melanin like pigment and are septate hyphae with pseudohyphae, sometimes co-existing with yeast forms [4]. The morphology of Phaeoacremonium genus is intermediate between Acremonium and Phialophora genus and all are pathogens implicated in opportunistic infections [3].

Crous et al. in 1996 had proposed a species of Phaeoacremonium parasiticum with distinctive microscopic features [8]. In humans infected with Phaeoacremonium, cutaneous or subcutaneous lesions $[7,9]$ is the most common, but there have been reported cases of onychomycosis, endophthalmitis, central nervous system involvement, endocarditis, and disseminated disease (fungemia) [4, 9]. Phaeoacremonium species reported to cause human infection have included $P$. parasiticum, $P$. rubrigenum, $P$. alvesii, P. krajdenii, P. amstelodamense, $P$. griseorubrum, $P$. tardicrescens, and P. venezuelense. Most are reported to be associated with solid organ transplants [10]. Although there are reports of the fungus in various microbiology journals, however there are very few reports of cytological detection in literature [4].

The relative rarity of this fungus has led to difficulty in the identification of Phaeoacremonium species in 


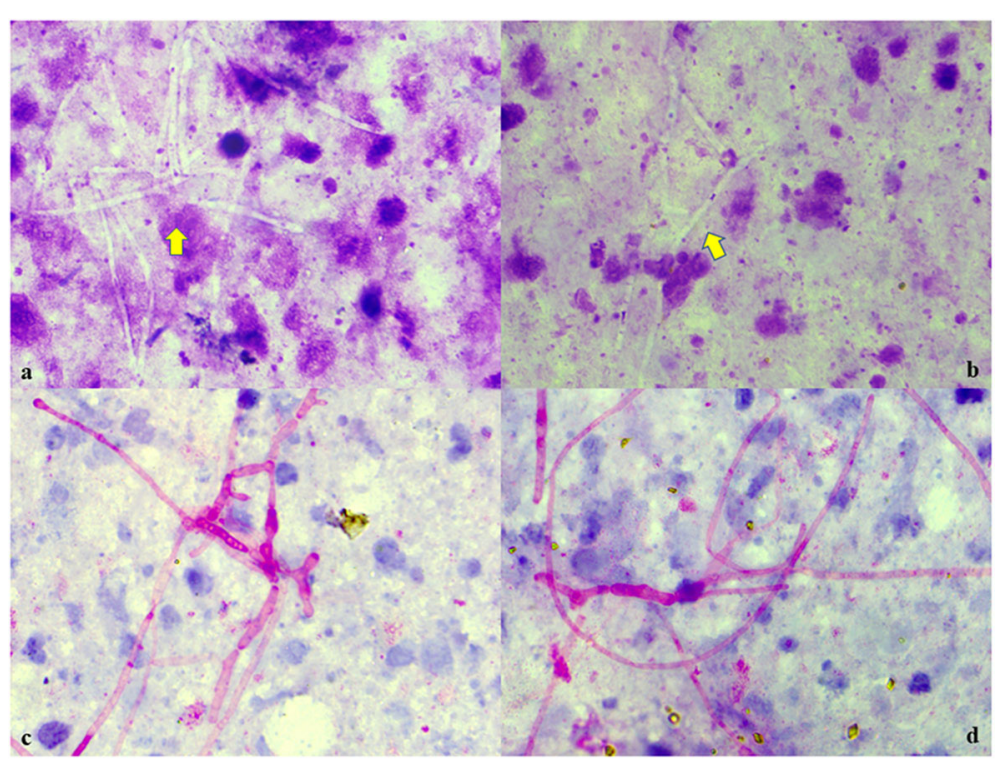

Fig. 3 a, b Cytosmears showing numerous fungal septate hyphae with acute angle branching along with plenty of degenerated inflammatory cells, histiocytes, nuclear debris, Splendore-Hoeppli phenomenon. (Geimsa, $\times 100$ ); c, d Periodic acid-Schiff (PAS) stain was positive for the fungal septate branching hyphae. (PAS, × 100)

cultural and microscopic fields [1]. Although difficult, fungal and microscopic features can be used for the identification of the Phaeoacremonium species as flat predominantly felty colonies with a woolen texture. These colonies are brown in color with varying shades of pale to dark brown. The mycelium has septate hyphae either singly or in bundles of fascicles. The classic morphologic characters are the combination of conidiophore, phialides, and allantoid conidia.

Molecular tools may be used based on the analysis of the $\beta$-tubulin gene sequencing by PCR, is available in limited centers [11]. Patients are usually rural workers with daily contact with soil, wood splinters, thorns, or other trauma-causing objects [7], so is our patient who is a daily laborer.

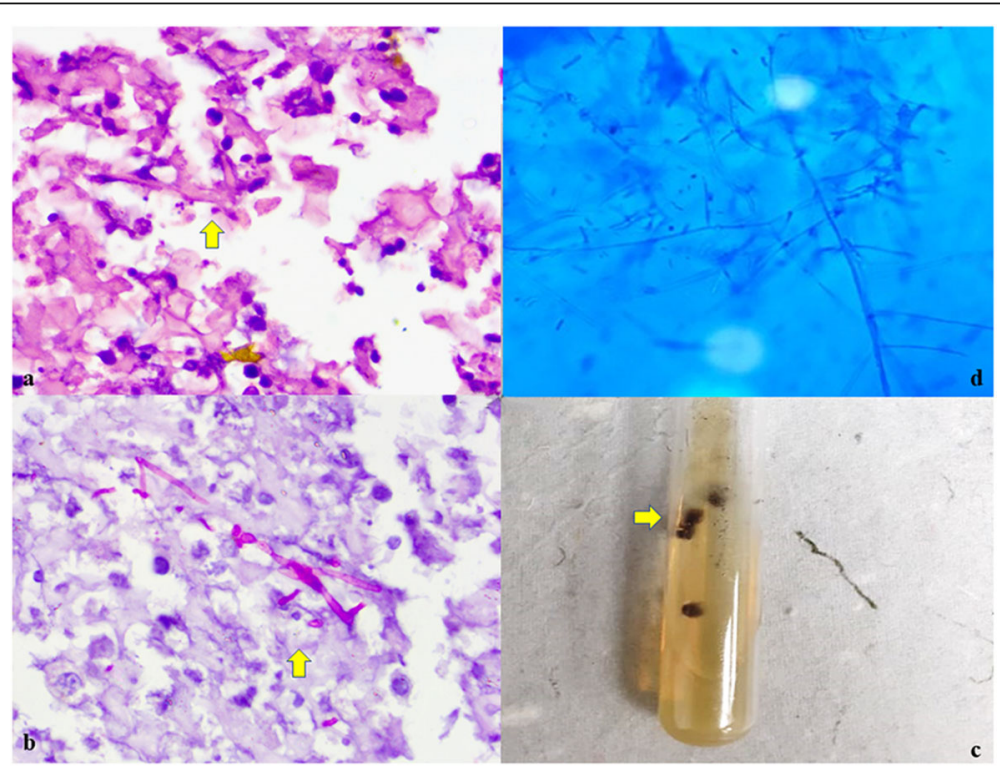

Fig. 4 a, b Microscopy showing numerous fungal hyphae with acute angle branching and inflammatory cells with PAS positivity. (H\&E, $\times 100$; PAS, $\times 100)$; c SDA slant showing surface greyish beige to black color and developed clusters of aerial hyphae; $\mathbf{d}$ Lactophenol cotton blue (LPCB) mount showing young hyphae forming thick bundles (fascicles), cylindrical phialides along the hyphae and oblong conidia. (LPCB, $\times 40)$ 
Fine needle aspiration is a basic and essential technique that is minimally invasive and used to investigate superficial or deep-seated lesions. Rapid on-site evaluation (ROSE) is an add on to the routine FNAC in providing a provisional diagnosis and collection of extra samples for cell block, special stains, fungal cultures, and molecular studies $[6,12]$. In our case also ROSE using $1 \%$ aq. toluidine blue had helped in arriving at the diagnosis.

In the absence of granuloma, giant cells, negative $\mathrm{ZN}$ stain we were able to exclude tuberculosis, sarcoidosis, giant cell tumor. Fungal morphology was helpful to exclude the possibility of Rhinosporidiosis \& Aspergillosis $[4,5]$. The other differential diagnosis can be chromoblastomycosis which are more superficial disease with sclerotic bodies, micro abscesses, and can be surgically managed [13].

There is no standardized treatment for these rare fungal infections. However, surgical wound excision can be combined with systemic Itraconazole therapy for several months [14]. Antifungals such as amphotericin, fluconazole, posaconazole, ketoconazole, terbinafine, and 5 -fluorocytosine $(5 \mathrm{FC})[15,16]$ may be for patients with severe disease, poor response or hepatic toxicity to itraconazole [4].

$P$. parasiticum is an emerging opportunistic fungal infection with limited knowledge regarding the clinical presentation, treatment protocol. A high index of suspicion with confirmation by cytological, histopathological, special stains, culture and molecular techniques for early appropriate treatment is required.

\section{Abbreviations \\ eAG: Estimated average glucose; FNAC: Fine needle aspiration cytology; LPCB: Lactophenol cotton blue; ROSE: Rapid on-site evaluation; PAS: Periodic acid Schiff; SDA: Sabouraud's dextrose agar; ZN: Ziehl-Neelsen; 5FC: 5 - fluorocytosine}

\section{Acknowledgements}

Dr. Ritu Nayar, Deputy HOD, Department of Microbiology, Dr. Lal path Labs, New Delhi for her technical support.

\section{Disclosure of grants}

Nil

\section{Authors' contributions}

ST carried out concepts \& design, literature search, participated in clinical study and will stand as guarantor also. BN carried out data acquisition, data analysis \& manuscript preparation. AS, AAB carried out concepts \& design, literature search. PB \& NK carried out concepts \& design, literature search. All the authors have read $\&$ approved the final manuscript.

\section{Funding}

$\mathrm{NIL}$

\section{Availability of data and materials}

All the data regarding the findings are available within the manuscript.

Ethics approval and consent to participate

Not applicable.

\section{Consent for publication}

Written consent for publication and any additional related information was taken from the patient involved in the study.

\section{Competing interests}

The authors declare that they have no competing interests.

\section{Author details}

'Department of Pathology, All India Institute of Medical Sciences, Mangalagiri, Andhra Pradesh 522503, India. ${ }^{2}$ Department of General Surgery, All India Institute of Medical Sciences, Mangalagiri, Andhra Pradesh 522503, India. ${ }^{3}$ Department of Microbiology, All India Institute of Medical Sciences, Mangalagiri, Andhra Pradesh 522503, India. ${ }^{4}$ Department of Radiology, All India Institute of Medical Sciences, Mangalagiri, Andhra Pradesh 522503, India.

Received: 25 July 2020 Accepted: 3 September 2020

Published online: 20 September 2020

\section{References}

1. Severo CB, Oliveira FM, Pilar FES, Severo CL. Phaeohyphomycosis: a clinicalepidemiological and diagnostic study of eighteen cases in Rio Grande do Sul, Brazil. Mem Inst Oswaldo Cruz. 2012;107(7):854-8 Rio de Janeiro.

2. Gramaje D, Mostert L, Roenewald JZ, Crous PW. Phaeoacremonium: from esca disease to phaeohyphomycosis. Fungal Biol. 2015;119:759-83.

3. Ajello L, Georg LK, Steigbigel RT, Wang CJ. A case of phaeohyphomycosis caused by a new species of Phialophora. Mycol. 1974;66:490-8.

4. Rajan STM, Bhaskaran S, George M, George SH. Phaeoacremonium parasiticum hand infection in renal transplant patient. J Acad Clin Microbiol. 2019;21(1):40-3.

5. Arora S, Khatter S, Agarwal C, Imaculata X. Cytological dilemma of a rare sub cutaneous fungal infection Phaeoacremonium griseorubrum. Indian J Case Rep. 2018;4(3):191-3.

6. Kanchan K, Santosh T, Agnihotri M, Sathe P, Naik L. This 'Rose' has no thorns - diagnostic utility of 'rapid on-site evaluation' (ROSE) in fine needle aspiration cytology. Indian J Surg Oncol. 2019;10(4):688-98.

7. Choi J, Lee Y, Chung HS, Koo JS, Yong D, Kim YS, et al. Subcutaneous Phaeohyphomycosis caused by Phaeoacremonium species in a kidney transplant patient: the first case in Korea. Korean J Lab Med. 2011;31:201-4.

8. Crous PW, Gams W, Wingfield MJ, Van Wyk P. Phaeoacremonium gen. nov. associated with wilt and decline diseases of woody hosts and human infections. Mycologia. 1996;88:786-96.

9. Furudatea S, Sasaia S, Numataa Y, Fujimurab T, Aibab S. Phaeohyphomycosis caused by Phaeoacremonium rubrigenum in an immunosuppressive patient: a case report and review of the literature. Case Rep Dermatol. 2012;4:119-24.

10. Mostert L, Groenewald JZ, Summerball RC, Robert V, Sutton DA, Padhye AA, et al Species of Phaeoacremonium associated with infections in humans and enviormnetal reservoirs in infected woody plants. J Clin Microbiol. 2005:43(4):1752-67.

11. Baddley JW, Mostert $L$, Summerbell RC, Moser SA. Phaeoacremonium parasiticum infections confirmed by $\beta$-tubulin sequence analysis of case isolates. J Clin Microbiol. 2006;44(6):2207-11.

12. Santosh T, Puneeta N, Patro MK, Gaikwad P. Fine needle aspiration as a diagnostic tool in cysticercosis: a case series. J Med Case Rep. 2019;13:92-6.

13. McGinnis MR. Chromoblastomycosis and phaeohyphomycosis: new concepts, diagnosis, and mycology. J Am Acad Dermatol. 1983;8(1):1-16.

14. Ogawa MM, Galante NZ, Godoy P, Fischman-Gompertz O, Martelli F, Colombo AL, et al. Treatment of subcutaneous phaeohyphomycosis and prospective follow-up of 17 kidney transplant recipients. J Am Acad Dermatol. 2009:61:977-85.

15. Badali H, Khodavaisy S, Fakhim H, de Hoog GS, Meis JF, Chowdhary A. In vitro susceptibility profiles of eight antifungal drugs against clinical and environmental strains of Phaeoacremonium. Antimicrob Agents Chemother. 2015;59:7818-22.

16. Monaganti S, Santos CAQ, Markwardt A, Pence MA, Brennan DC. Pulmonary phaeohyphomycosis caused by Phaeoacremonium in a kidney transplant recipient: successful treatment with posaconazole. Case RepMed. 2014:5. Article ID 902818. https://doi.org/10.1155/2014/902818.

\section{Publisher's Note}

Springer Nature remains neutral with regard to jurisdictional claims in published maps and institutional affiliations. 